\title{
REVISIONE ARTICOLO
}

SOUZA, Jeane Mendes Pinheiro de ${ }^{[1]}$

SOUZA, Jeane Mendes Pinheiro de. Depressione tra i docenti in Brasile: una revisione della letteratura. Revista Científica Multidisciplinar Núcleo do Conhecimento. Anno 06, Ed. 06, Vol. 12, pp. 128-141. Giugno 2021. ISSN: 2448-0959, Link di accesso: https://www.nucleodoconhecimento.com.br/psicologia-it/depressione-tra-i-docenti, DOI: 10.32749/nucleodoconhecimento.com.br/psicologia-it/depressione-tra-i-docenti

Contents

- RIEPILOGO

- 1. INTRODUZIONE

- 2. DEPRESSIONE

- 2.1 MALINCONIA: UNA FORMA DI DEPRESSIONE

- 2.3 TRISTEZZA E DEPRESSIONE

- 3. DEPRESSIONE TRA GLI INSEGNANTI

- 3.1 CONTESTO E CAUSE

- 3.2 EFFETTI E CONSEGUENZE

- 3.3 PERCORSI E ALTERNATIVE PER FAR FRONTE ALLA DEPRESSIONE

- 3.4 PROPOSTE D'AZIONE DA ADOTTARE

- 4. CONSIDERAZIONI FINALI

- RIFERIMENTI

RIEPILOGO

Al giorno d'oggi, i problemi di salute che comportano stress legato al lavoro sono una realtà sempre più comune. In questo contesto, ci sono professioni e professionisti che sono più esposti e soggetti a questo tipo di patologie, come i professionisti dell'istruzione. Studi condotti in tutto il mondo indicano che gli educatori sono a grande rischio di soffrire di esaurimento fisico e/o mentale, che comportano le difficoltà materiali e psicologiche associate all'esercizio dell'attività didattica. È noto che tali problemi di salute compromettono 
notevolmente la qualità della vita di questi professionisti e, tra questi, spicca la depressione nelle sue manifestazioni che possono comportare malinconia e tristezza in varie forme e a vari livelli. L'impegno per la qualità della vita di questi professionisti rende sempre più necessario approfondire ricerche e approcci che possano collaborare al fine di trovare alternative che minimizzino gli effetti di questo male. A causa di quanto sopra, questo lavoro mira a condurre uno studio sulla depressione e il suo verificarsi tra gli insegnanti. Come metodologia, è stata condotta una ricerca bibliografica con una rassegna di studi e autori che approfondiscono l'approccio alle materie depressione, depressione tra gli insegnanti, malinconia e alternative per far fronte alla depressione.

Parole chiave: Depressione, Qualità della vita, Malinconia, Tristezza, Professionisti dell'Istruzione.

\section{INTRODUZIONE}

Ogni volta, secondo la cultura e i costumi di ogni società, emergono nuove malattie e patologie che colpiscono individui e gruppi sociali. Ciò è dovuto alla natura del comportamento e delle cariche a cui sono sottoposte le persone, dovuta ai valori e alle esigenze evidenziati in ogni epoca. Così, anche le professioni che hanno una lunga storia, riconosciute per la loro importanza, possono essere influenzate dalle riflessioni dei cambiamenti nei valori e nel comportamento.

Così, si trova come una realtà attuale l'alta incidenza di casi di depressione nella società nel suo complesso e, più specificamente, tra le professioni che richiedono un grande sforzo, a causa dello stress che rappresentano, come l'insegnamento. È noto che c'è un'alta incidenza di depressione tra gli insegnanti, una malattia vista come il male del secolo e la quarta causa mondiale di malattia e che colpisce le persone delle più svariate culture, fasce d'età e classi sociali (AROS, 2008).

Una delle cause di depressione tra gli insegnanti è che il lavoro viene eseguito in ambienti contrastanti con un elevato carico di requisiti di lavoro, tra cui spiccano le molte attività extra-classe, riunioni e attività aggiuntive. Inoltre, i problemi con gli studenti che possono raggiungere minacce e aggressioni verbali e fisiche sono molto comuni. Questa realtà 
stressante influisce sulla salute fisica e mentale e sul rendimento professionale degli insegnanti (CAPEL, 1987).

È importante sottolineare che, in questo contesto, quando l'insegnante si ammala, la scuola ha compromesso la sua funzione sociale che alla fine non si materializza. Pertanto, la formazione dei cittadini e la costruzione dell'autonomia e la consapevolezza della cittadinanza sono compromesse. La depressione nei suoi gradi più vari è stata considerata una delle malattie che fanno parte del quadro della sindrome dell'esaurimento professionale (Burnout) (MINISTÉRIO DA SAÚDE, 2001).

Considerando l'importanza del problema e la necessità di discutere gli aspetti più rilevanti sull'argomento, questo lavoro mira a condurre uno studio sulla depressione e sul suo verificarsi tra gli insegnanti. Come metodologia, è stata condotta una ricerca bibliografica con una rassegna di studi e autori che approfondiscono l'approccio alle materie depressione, depressione tra gli insegnanti, malinconia, tristezza e alternative per far fronte alla depressione.

\section{DEPRESSIONE}

Secondo l'Organizzazione Mondiale della Sanità (OMS, 2002) la depressione è un disturbo mentale comune, che ha come caratteristiche "tristezza, perdita di interesse, mancanza di piacere, oscillazioni tra sensazione di colpa e bassa autostima, oltre a disturbi del sonno o dell'appetito" e "sensazione di stanchezza e mancanza di concentrazione". Ė degno di nota il fatto che il grado di disabilità accreditato ai disturbi depressivi sia più alto che in altre malattie fisiche croniche, come ipertensione, diabete mellito $o$ artrite.

I tipi accidentali o reazionari di depressione motivati sono qualcosa che potrebbe aver modificato o trasformato la vita di una persona in qualche modo. Quella che viene convenzionalmente chiamata vita depressiva si verifica quando la depressione è permanente, che può essere vista nelle persone che mostrano sempre una sensazione di disagio. In questo caso, i pochi momenti in cui stanno bene sono passeggeri, non costituendo una solida base per la vita (TATOSSIAN, 2012).

Nella depressione ci sono diverse manifestazioni che configurano questo problema. La 
tristezza può essere considerata un sintomo di condizioni depressive ed esprime una manifestazione esteriore di problemi derivanti dalla psiche. Questo tipo di manifestazione non è particolarmente evidenziato, perché ha nella sua rilevanza il grado di occorrenza e permanenza che varia notevolmente. Tuttavia, può compromettere la qualità della vita quando si verifica frequentemente (FUCHS, 2013).

Per quanto riguarda la malinconia, differisce dalla tristezza a causa dei diversi livelli che occupano nella vita psichica del soggetto. La malinconia "è caratterizzata come un disturbo dell'ordine dell'umorismo e non del sentimento, come la tristezza. il sentimento è un'azione, un movimento affettivo e, quindi, ha una direzione verso qualsiasi cosa o qualcuno" (TATOSSIAN, 2012).

\subsection{MALINCONIA: UNA FORMA DI DEPRESSIONE}

Il termine malinconia è usato secondo il Manuale diagnostico e statistico dei disturbi mentali (DSM IV) per determinare un sottotipo di depressione. A differenza di altre forme di male, la depressione malinconica può avere come fattore determinante gli aspetti genetici sarebbero $i$ principali determinanti. Le alterazioni psicomotorie si distinguono in questo tipo di depressione (LAFER et al., 1996).

Secondo Tatossian (2006, p. 113), la depressione malinconica si verifica quando c'è, nelle parole dell'autore, "un'esperienza di emurcheamento e delinof the lived, la perdita di freschezza". La sensazione di malinconia è responsabile della sofferenza dovuta all'isolamento vissuto dal soggetto. In questo contesto, è importante differenziare la sofferenza normale da una sofferenza malinconia, perché la malinconia causa più di quanto si possa esprimere con episodi di tristezza, che sono solo una delle forme di espressione della malinconia.

Allo stesso tempo, la malinconia si manifesta come una delle forme di depressione, avendo nella sua causa una disfunzione neurochimica che può essere corretta dall'azione dei farmaci psicotropi. Secondo Freud, il termine malinconia sarebbe usato come riferimento a uno stato depressivo più intenso e più grave, in cui sono stati aggiunti diversi elementi. La parola malinconia sarebbe usata per definire un'immagine con una predominanza di intensi effetti 
depressivi (DELOUYA, 2002, p. 22).

\subsection{TRISTEZZA E DEPRESSIONE}

La tristezza e la depressione sono molto comunemente confuse, tuttavia, differiscono, perché c'è un'eventualità legata alla tristezza, poiché la depressione si verifica in un contesto di una certa costanza di questa tristezza. "La tristezza fa parte della condizione umana, così come molti altri sentimenti. Si può essere sconvolti perché quel giorno non c'era produzione di ciò che si voleva, o anche a causa di una discussione, ma la tristezza passa. D'altra parte, quando si tratta di depressione, c'è un maggiore coinvolgimento o compromissione di altre aree, come il comportamento, la psicomotricità, l'espressione del pensiero e la cosiddetta neurovegetativa, legata al sonno e all'alimentazione. Quando si tratta della diagnosi di depressione, si cerca due sintomi di base: "quello che viene chiamato umore depresso, che è questa tristezza, disperazione, vuoto, angoscia, a volte piangendo, persino un'irritabilità; e la perdita o la diminuzione dell'interesse o del piacere nelle cose della vita" (SENE-COSTA, 2011, p. 37).

\section{DEPRESSIONE TRA GLI INSEGNANTI}

Secondo l'Organizzazione internazionale del lavoro (OIT), il ruolo degli insegnanti nella società è essenziale per la formazione del cittadino a vita. A causa della natura del loro lavoro, questi professionisti hanno una grande responsabilità, ma, d'altra parte, non ci sono idee sulle condizioni di lavoro che possano contribuire alla sicurezza e alla salute di questi professionisti (OIT, 1984).

Tra i problemi di salute che hanno colpito questi professionisti, spicca la depressione. Tra i fattori che contribuiscono all'emergere di questo problema, "il lavoro informativo, l'indisciplina in classe, il basso salario, la violenza, le richieste dei genitori degli studenti, il bombardamento di informazioni, l'esaurimento fisico e, soprattutto, il mancato riconoscimento della loro attività" (GASPARINI et al., 2005).

Queste informazioni sono corroborate da studi che dimostrano questi eventi, come i seguenti, nel rapporto D'Agostini (2019): 
Secondo un sondaggio dell'Organizzazione per la cooperazione e lo sviluppo economico (OCDE) con oltre 100.000 insegnanti e presidi di scuole nel secondo ciclo della scuola elementare, il Brasile è il Paese con il più alto tasso di violenza contro gli insegnanti.L'indagine è stata condotta con 34 paesi e ha rilevato che il $12,5 \%$ degli insegnanti brasiliani è stato vittima di aggressioni verbali o intimidazioni nei confronti degli studenti almeno una volta alla settimana (D'AGOSTINI, 2019).

Quando si considerano le condizioni di lavoro, le circostanze in cui gli insegnanti devono usare le loro capacità fisiche, cognitive e affettive per agire nella formazione delle persone, questa responsabilità può portare a uno sforzo molto maggiore di quello che dovrebbe essere impiegato dagli insegnanti, il che innesca uno stress, che è una delle principali cause di depressione (GASPARINI , 2005).

\subsection{CONTESTO E CAUSE}

Al giorno d'oggi, il ruolo che l'insegnante svolge va oltre quanto ci si aspetta nella mediazione del processo di conoscenza dello studente. II rendimento del docente ha superato i limiti dell'aula in modo che fosse possibile stabilire una comunicazione tra la scuola e la comunità scolastica. Pertanto, l'insegnante, oltre all'insegnamento, è responsabile della partecipazione alla gestione e alla pianificazione della scuola. Pertanto, deve dedicare di più, anche nelle sue responsabilità alle famiglie e alla comunità.

La complessità dei compiti che gli insegnanti devono spesso prendere in considerazione mezzi di riqualificazione e il tempo necessario per acquisire le conoscenze necessarie. Così, gli educatori finiscono per dover cercare alternative per se stessi che si traducono in un aumento non riconosciuto e non retribuito dell'orario di lavoro (OLIVEIRA, 2003).

Uno studio condotto in diverse località, come Belo Horizonte e Montes Claros (MG), Vitória da Conquista e Salvador (BA), Santa Maria (RS) e Campinas (SP), tra gli altri, con l'obiettivo di verificare le condizioni di salute dell'insegnante, ha indicato un'alta incidenza di domande di congedo medico e le loro motivazioni. Sono state evidenziate come motivazioni, aspetti legati alle condizioni di lavoro: tra gli altri, "compiti eccessivi e rumore, pressione per la 
riqualificazione professionale, mancanza di sostegno istituzionale e insegnanti in numero necessario". Lo studio sottolinea anche che tali problemi "generano uno sforzo troppo sforzo nello svolgimento dei loro compiti". Le conclusioni mostrano che "i risultati misurati nelle varie città sono convergenti e che gli insegnanti sono più soggetti di altri gruppi ad avere disturbi psichici di varia intensità" (CURI, 2010).

I professionisti dell'istruzione si sentono sotto pressione Molti di questi elementi di pressione sono il risultato di una riconfigurazione del mondo del lavoro, che non è stata effettuata con soddisfazione per quanto riguarda il soddisfacimento delle esigenze dell'insegnante nella stessa scala su cui è caricato. Il sistema scolastico trasferisce al professionista la responsabilità di colmare le lacune dell'istituto, che stabilisce meccanismi rigidi e ridondanti di valutazione professionale, afferma Sandra Gasparini (CURI, 2010).

A causa della svalutazione della carriera didattica, si è registrato un significativo aumento dei problemi psichici tra gli insegnanti a causa del cambiamento di valori e della crescita della chiamata al consumo e dell'apprezzamento di dover a scapito dell'essere. In un'indagine condotta nel 2003 dall'Unione degli insegnanti dell'istruzione ufficiale dello Stato di San Paolo (Apeoesp), è stata evidenziata una percentuale del " $46 \%$ degli insegnanti che hanno già diagnosticato un qualche tipo di stress, raggiungendo una percentuale del $51 \%$ tra le donne (CURI, 2010).

Secondo la Confederazione nazionale degli operatori dell'istruzione (CNTE), nel Mato Grosso do Sul, "oltre il $60 \%$ dei congedi medici concessi agli operatori dell'istruzione nello stato è per gli insegnanti. Sul numero totale di licenze, il 38\% è correlato a disturbi mentali e comportamentali, la ragione principale delle assenze" (CURI, 2010).

Un altro motivo di stress per gli insegnanti è la costante paura di perdere il lavoro tra gli insegnanti di istituti privati: in alcuni (istituti), gli studenti si ribellano all'insegnante e la scuola preferisce licenziare il professionista, anche senza una vera ragione. Nelle scuole pubbliche, la costante pressione per numeri che indicano alte percentuali di approvazione, a causa della valutazione delle prestazioni e della meritocrazia adottata dalle amministrazioni di alcune città e stati, viene imposta una pressione sugli insegnanti. Questa insicurezza può contribuire in modo decisivo alla depressione tra gli insegnanti, che può verificarsi, sia tra i professionisti che lavorano nelle scuole pubbliche, sia tra gli insegnanti del sistema scolastico 
privato (CURI, 2010).

\subsection{EFFETTI E CONSEGUENZE}

Lo stress e la depressione legati agli eventi durante le routine lavorative sono comuni per i professionisti dell'istruzione. Esempi di ciò sono gli eventi dopo episodi di violenza contro gli educatori. È possibile ricordare gli eventi del 2018 registrati presso il Dipartimento Comunale dell'Istruzione della città di Rio de Janeiro che "hanno rilasciato 3.055 licenze per malattie legate allo stress e alla depressione", dove "un insegnante è autorizzato ogni tre ore per malattie legate allo stress" (D'AGOSTINI, 2019).

La violenza nelle scuole può avere impatti sulla salute che possono portare a effetti più lunghi, come la depressione, ma possono anche derivare dalla condizione depressiva preesistente stessa. I principali impatti della violenza scolastica sulla salute sono considerati "sintomi psicosomatici come mal di testa, vertigini, nausea, diarrea, enuresi, sudorazione, aquicardia, dolore muscolare, cambiamenti del sonno (insonnia o sonno eccessivo)"; conseguenze dello "stress" che può aumentare la vulnerabilità alle malattie diminuendo la resistenza immunitaria; problemi psicologici "come ansia, paura, rabbia, irritabilità, irrequietezza, stanchezza, insicurezza, isolamento, impotenza, rifiuto, tristezza, angoscia, bassa autostima, depressione e pensieri suicidi, tra gli altri; problemi di socializzazione, con l'aumento dell'"isolamento sociale, generando insicurezza che può incidere sulla fiducia nell'altro, la capacità di esprimersi in pubblico, di risolvere i conflitti e prendere decisioni" (D'AGOSTINI, 2019).

\subsection{PERCORSI E ALTERNATIVE PER FAR FRONTE ALLA DEPRESSIONE}

Secondo gli operatori sanitari coinvolti nel trattamento della depressione, ci sono alternative e percorsi che differiscono, il che dipende dal grado di usura e profondità del quadro di depressione. È possibile, in alcuni casi, che la persona possa sottoporsi solo a psicoterapia; può essere temporaneamente medicato con ansiolitico o antidepressivo, in concomitanza con la psicoterapia, e a volte ci sono casi in cui il professionista deve essere trasferito a una funzione burocratica o deve lavorare con un altro profilo di studenti (D'AGOSTINI, 2019). 
È importante sottolineare che ci sono progetti che possono essere adottati da scuole e reti educative, come quello implementato in una scuola di Ribeirão Preto, SP, che mirava a "sviluppare l'autostima, la tolleranza e la cooperazione tra gli studenti". Il progetto è stato adottato a causa della presentazione da parte della scuola di un "alto tasso di violenza sul posto e difficoltà nel processo di insegnamento-apprendimento". L'idea è "di adottare misure preventive contro la violenza che, pur focalizzate sugli studenti, intasano il clima scolastico nel suo complesso e promuovano positivamente una maggiore salute mentale negli istituti scolastici" (D'AGOSTINI, 2019).

Anche altre azioni che possono essere efficaci sono la riflessione "sui fattori che portano allo stato depressivo degli insegnanti attraverso le narrazioni degli individui stessi. Questo permette di capire "come l'insegnante vive la malattia, quali sono le loro aspettative sul superamento del problema e soprattutto e come l'insegnamento viene rassegnato dopo il congedo". Questa comprensione consente di sviluppare programmi per "identificare, assistere e trattare i professionisti colpiti dalla depressione, nonché per aiutare i manager e i professionisti dell'istruzione a identificare i segni del corpo, i sintomi e i comportamenti caratteristici della malattia" (SOUZA e SOUZA, 2015, p. 182).

Condividendo esperienze e avendo contatti con altre narrazioni, il professionista dell'istruzione può ripensare la propria traiettoria, che può evitare un allontanamento dalla professione. È anche possibile analizzare le cause dello stress nell'insegnamento, rendendo possibile per gli educatori "vedere quali problemi contribuiscono alla malattia nella loro professione e come minimizzare questi problemi" (SOUZA e SOUZA, 2015, p. 182).

\subsection{PROPOSTE D'AZIONE DA ADOTTARE}

Si consiglia di adottare un animale domestico come un modo per affrontare gli effetti della depressione. Con la relazione che si afferma a causa dell'interazione che è necessaria e che si verifica anche spontaneamente, è necessario svolgere attività che aumentano i livelli di serotonina e dopamina, che sono legati alla sensazione di benessere. L'attenzione e la cura che sono incoraggiate dal desiderio di promuovere il benessere dell'animale domestico causano, in modo naturale, attività fisiche, come camminare o giocare, che rilasciano endorfine che alleviano i sintomi della malattia. Secondo gli esperti, la vicinanza di un 
animale domestico può scongiurare pensieri negativi e ridurre l'angoscia, la tristezza e la solitudine (REED et al., 2012).

La depressione dovuta al suo carattere di inattività può e deve essere affrontata da attività che stimolano coloro che soffrono di questo male. Pertanto, la danza può agire per ridurre i sintomi della depressione e dell'ansia. Ballare significa che può verificarsi l'espressione dei sentimenti e il miglioramento di un'auto-percezione delle emozioni, che può anche contribuire al benessere fisico e sociale delle persone che soffrono di questo male. $\grave{E}$ anche noto che la danza può avere effetti positivi sull'umore, oltre a un miglioramento della capacità aerobica, della flessibilità e della capacità di percepire il corpo, che può portare a un miglioramento dell'autostima che di solito è compromesso a causa della depressione. Ballare migliora la conoscenza di sé e stimola l'apprezzamento del proprio corpo e la capacità che dimostra di espressione (SILVA, 2015).

Un altro atteggiamento da adottare è il salvataggio della percezione del piacere nelle cose semplici e nei momenti della vita quotidiana. L'idea è che si possa percepire il significato e il piacere nelle azioni, negli eventi e negli eventi della vita quotidiana in modo che si possa anche valore ciò che è più grande. Si dovrebbe iniziare ad osservare ciò che è bello e che risveglia questa percezione di ciascuno e che può variare in molto, fiori, momenti della giornata (alba o tramonto), il gusto di un cibo, in piccole astrazioni che funzioneranno in modo diverso per ciascuno, ma che avranno lo stesso effetto. L'idea, per chi già presenta una condizione depressiva, è quella di "rilevarsi per concentrarsi su ciò che non può essere realizzato a causa della malattia" (GAUER, 1997).

Un'altra misura da adottare è quella di impegnarsi in attività che forniscono piacere, che può anche variare notevolmente, a seconda di ogni persona. per coloro che hanno già depressione, si dovrebbe "ricominciare a svolgere attività che erano una volta divertenti". $\dot{E}$ importante determinare, tuttavia, ciò che suscita interesse al momento, che potrebbe essere cambiato. Se necessario, dovrebbero essere ricercate nuove forme di divertimento. Questo può essere stimolato da persone con cui vivono o da sole coloro che soffrono di depressione, senza rinunciare a cercare qualche attività piacevole, anche se non funziona in pochi istanti. È importante cercare "novità, imparare cose nuove e piacevoli", che eviti il fallimento delle azioni (GAUER, 1997). 
Un'altra misura essenziale per le persone che soffrono di depressione è un sonno equilibrato e sano perché, in genere, queste persone dormono troppo o non possono dormire. Secondo gli esperti ciò è dovuto "ai cambiamenti nei livelli di serotonina e noradrenalina, ormoni che regolano il sonno. Poiché "il sonno è essenziale per il cervello per regolare nuovamente questi ormoni e mitigare gli effetti della depressione", dovrebbe verificarsi in equilibrio senza esagerare o assenza. Per coloro che non hanno sonno, gli esercizi di respirazione sono indicati per il rilassamento e la facilitazione quando si addormentano. Se la persona dorme troppo, è indicato che viene risvegliata e stimolata in qualche modo in modo che non dormi eccessivamente (GAUER, 1997).

\section{CONSIDERAZIONI FINALI}

Si osserva come una realtà della società contemporanea che gli insegnanti dimostrano di avere una maggiore propensione alla sofferenza psichica tra le quali spicca la depressione nelle sue fasi o la sua scala di gravità. Analizzando i rapporti tra salute e lavoro, è possibile percepire le associazioni tra i problemi identificati e le condizioni e le caratteristiche del lavoro descritto.

I professionisti dell'istruzione, essendo esposti ad alti livelli di stress, che si verificano per diversi motivi, finiscono vittimizzati da depressione che si presenta in vari modi e può raggiungere livelli che finiscono per portare il professionista a lasciare il lavoro, senza capire cosa lo motiva, senza il quale può diventare impossibile restituire il professionista per svolgere la professione per la quale è stato formato e unico con cui è stato formato e unico con cui è stato spesso ha esperienza.

Molti professionisti dell'istruzione si trovano immotivati per il lavoro, ma non hanno altra alternativa che continuare, perché ne hanno bisogno per il loro sostentamento. La stragrande maggioranza non può perdere tempo investito nella propria formazione e nelle esperienze professionali uniche accumulate nel corso di una carriera professionale e continuare, anche senza condizioni minime per lo svolgimento del lavoro. Ciò va a scapito di tutti: professionisti e società nel suo complesso.

Pertanto, è essenziale che vi sia apprezzamento per i professionisti e l'accoglienza di 
istituzioni che dovrebbero ascoltare e fornire momenti in modo che i professionisti possano esprimere le loro paure, malcontenti e sentimenti. I professionisti dell'istruzione alternativa dovrebbero essere offerti per avvicinarsi a coloro che sono già malati e profilassi per coloro che potrebbero ammalarsi. È noto che ci dovrebbero essere cure per coloro che sono già in depressione, ma il reinserimento di questi professionisti comporta la necessità che siano accolti, ascoltati e guidati.

RIFERIMENTI

AROS, M.S. Produção científica sobre depressão: Análises de resumos. Campinas, SP. Universidade Católica de Campinas, 2008.

CAPEL, S.A. The incidence of and influences on stress and burnout in secondary school teachers. Br J Educ Psychol 1987; 57:279-88.

CARLOTTO, M.S. Burnout e o trabalho docente: considerações sobre a intervenção. Revista Eletrônica InterAção Psy 2003; 1:12-8.

CURI, F. O professor brasileiro está doente. Revista Educação - Edição 119, 2010. Disponível em: $\quad<$ http://stellabortoni.com.br/index.php/artigos/1177-o-paofissoa-baasiliiao-ista-ioioti>. Acesso em: 17 de Jan. de 2021.

D'AGOSTINI, A. C. Brasil lidera índice de violência contra professores: o que podemos fazer? Nova Escola, Junho/2019. Disponível em:< https://novaescola.org.br/ conteudo/17609/brasillidera-indice-de-violencia-contra-professores-o-que-podemos-fazer>. Acesso em: 10 de jan. de 2021.

DELOUYA, D. Depressão estação psique. São Paulo: Escuta - Fapesp, 2002.

FUCHS, T. Depression, intercorporeality, and interaffectivity. Journal of Consciousness Studies, 20(7-8), 219-38, 2013.

GASPARINI, S. M.; BARRETO, S. M.; ASSUNÇÃO, A. A. O professor, as condições de trabalho e os efeitos sobre sua saúde. Educ. Pesqui. São Paulo, v. 31, n. 2, p. 189-199, Ago. 2005. 
Disponível em: <http://www.scielo.br/scielo. php?script $=$ sci_arttext\&pid $=\$ 151797022005000200003 \& / n g=e n \& n r m=i s 0>$. Accesso em: 19 Jan. 2021.

GAUER, G. et al. Terapias alternativas: uma questão contemporânea em psicologia. Psicol. cienc. prof., Brasília, v. 17, n. 2, p. 21-32, 1997. Disponível em:<http://pepsic.bvsalud.org/scielo.php?script=sci_arttext\&pid=S141498931997000200004 $\& \mid n g=p t \& n r m=i s o>$. Acesso em: 18 de jan. 2021.

LAFER, B.; NIERENBERG, A. A.; ROSENBAUM, J. F.; FAVA, M. Outpatients with DSM-III-R versus DSM IV Melancholic Depression. Comprehensive Psychiatry 1996;37(1):37-9.

MINISTÉRIO DA SAÚDE. Doenças relacionadas ao trabalho: manual de procedimentos para os serviços de saúde. Brasília: Ministério da Saúde, 2001.

MOREIRA, A. G. C. Clínica da melancolia. São Paulo: Escuta - Edufpa, 2002.

OLIVEIRA, D. A. As reformas educacionais e suas repercussões sobre o trabalho docente. In Reformas educacionais na América Latina e os trabalhadores docentes. Autêntica: Belo Horizonte, 2003, p. 13-35.

ORGANIZAÇÃO INTERNACIONAL DO TRABALHO. A condição dos professores: recomendação Internacional de 1966, um instrumento para a melhoria da condição dos professores. Genebra: OIT/ Unesco, 1984.

ORGANIZAÇÃO MUNDIAL DA SAÚDE (OMS). Relatório Mundial de Saúde. Lisboa: Climepsi Editores, 2002.

REED, R.; FERRER, L.; VILLEGAS, N. Curadores naturais: uma revisão da terapia e atividades assistidas por animais como tratamento complementar de doenças crônicas. Rev. Latino-Am. Enfermagem. maio-jun. 2012.

SENE-COSTA, Elizabeth. As emoções e os transtornos depressivos. O teatro do perdão como palco de resgates. Rev. bras. psicodrama, São Paulo , v. 19, n. 2, p. 85-99, 2011.Disponível em: $<$ http://pepsic.bvsalud.org/scielo.php?script=sci_arttext\& 
pid $=S 010453932011000200008 \&$ Ing $=p t \& n r m=i s o>$. Acesso em: 02 jan. 2021.

SILVA, V. A. Benefícios afetivo-sociais da prática da dança para estudantes dos Núcleos de Arte da Prefeitura do Rio de Janeiro. Tese de Doutoramento. Faculdade de Motricidade Humana, Universidade de Lisboa, 2015.

SOUZA, E. C. Conte sua história: doença, crônica, narrativas e reconstrução biográfica. Curitiba: CRV, 2016.

TATOSSIAN, A. A fenomenologia das psicoses. São Paulo, SP: Escuta, 2006.

TATOSSIAN, A. Fenomenologia da depressão. In TATOSSIAN, A.; MOREIRA, V. Clínica do Lebenswelt: Psicoterapia e psicopatologia fenomenológica. São Paulo, SP: Escuta, 2012.

${ }^{[1]}$ Post-laurea in Terapia Cognitivo Comportamentale da UNINTER; Laureato in Psicologia presso Cesupi Faculdade de Ilhéus.

Inviato: Marzo 2021.

Approvato: Giugno 2021. 